\title{
Baseline thrombocytopenia complicated by recurrent episodes of transient severe thrombocytopenia following infections in an adult woman with a non involuting congenital hemangioma - a case report
}

\author{
Mitrakrishnan C Shivanthan ${ }^{1 *}$, Bandula Wijesiriwardena ${ }^{1,2}$ and Indira S Wijesiriwardena ${ }^{3}$
}

\begin{abstract}
Background: Congenital hemangiomas are benign abnormal proliferation of blood vessels. Noninvoluting congenital hemangiomas are a rare variant which persist, and may become bigger. Hemangiomas are known to be associated with thrombocytopenia, microangiopathic hemolytic anemia and Kasabach-Merritt phenomenon. Kasabach-Merritt phenomenon is characterized by consumptive coagulopathy with microangiopathic haemolyic anemia and thrombocytopenia. Platelet sequestration in the hemangioma or increased destruction which may either be immune or non immune are also further contributors to thrombocytopenia.

Case presentation: A 45 year old female with a non involuting hemangioma and baseline thrombocytopenia was observed to develop repeated episodes of transient severe thrombocytopenia associated with a variety of infectious conditions. Laboratory investigations suggested a peripheral mechanism. Platelet counts always returned to baseline levels on resolution of the precipitating infection.

Conclusion: The authors report this phenomenon as the first reported case of baseline thrombocytopenia complicated by recurrent episodes of transient severe thrombocytopenia following infections associated with a non involuting congenital hemangioma. The observations made in this patient were unique and hitherto unreported in medical literature. Both peripheral sequestration and destructive consumption were considered likely. Consumptive mechanisms were likely to encompass either or both immune and non immune causes. Further studies are needed to establish the precise pathogenesis.
\end{abstract}

Keywords: Hemangioma, Thrombocytopenia, Severe thrombocytopenia, Infection, Platelet

\section{Background}

Congenital hemangiomas are benign abnormal proliferation of blood vessels [1]. Noninvoluting congenital hemangiomas are a rare variant of cutaneous vascular tumors of intrauterine onset which persist - either unchanged or with slight enlargement [2]. Hemangioma-thrombocytopenia syndrome associated with microangiopathic hemolytic anemia has been documented in children. The KasabachMerritt phenomenon was described first in 1940 as a

\footnotetext{
* Correspondence: mcs627@gmail.com

'National Hospital of Sri Lanka, Regent Street, Colombo 7, Sri Lanka Full list of author information is available at the end of the article
}

syndrome of consumptive coagulopathy, in patients with capillary hemangiomas characterized by microangiopathic haemolyic anemia and thrombocytopenia [3]. Rapidly involuting congenital haemangiomas are known to be associated with fluctuating thrombocytopenia and coagulopathy [4]. Thrombocytopenia is a complication seen in adult patients with large or multiple hemangiomas [5]. This phenomenon may occur due to sequestration in the hemangioma or increased destruction which may be due to either immune or non immune causes [6]. Chronic immune thrombocytopenia is known to occur in infections [7], and acute immune mediated thrombocytopenia 
has been demonstrated in certain infections and hypersensitivity states [8-11]. Thrombocytopenia associated with infections has been known to parallel the severity of illness [12]. However literature search yielded no similar case with baseline thrombocytopenia complicated by recurrent episodes of transient severe thrombocytopenia following infections in a patient with a non involuting congenital hemangioma.

\section{Case presentation}

A 45 year old single woman with a non regressing congenital vascular malformation of the right arm and upper chest, presented with a 3 day history of high fever, chills, dysuria, hematuria and right loin discomfort. Her urine output was normal and she had no spontaneous bleeding manifestations or features of uremia. A marked drop in her platelet counts compared to recent baseline levels had been detected by her primary care physician following the onset of symptoms.

Her past medical history was significant for persistent low baseline platelet counts in the region of $100,000 / \mathrm{mm} 3$ with episodic reduction to values as low as $30,000 / \mathrm{mm} 3$ following infections. She had been extensively and repeatedly investigated from the time the low baselines platelet counts were detected at the age of 28 , however no definite cause was identified. On direct questioning she denied spontaneous mucocutaneous bleeding in the past. However her past medical history was significant for an abandoned plastic surgical procedure after developing a haematoma surrounding the preoperative intravenous cannulation site with a corresponding platelet count of $47,000 / \mathrm{mm} 3$. She had also been managed conservatively for uterine adenomyosis with depot provera from the age of 45 due to concerns of high risk of perioperative bleeding.

Detailed evaluation was negative for symptoms attributable to anemia, connective tissue disease and liver disease. History of medications was negative for agents associated with thrombocytopenia.

Examination revealed an ill looking lady with a temperature of 100.4 degrees Fahrenheit. She had a giant hemagioma involving the entire right arm, right lateral aspect of the neck and both the anterior and posterior aspect of the chest extending up to the 4th intercostal space (Figure 1). The hemangioma had small areas which were ulcerated and inflamed as well as healed scars. A soft bruit was appreciated on auscultation over the hemangioma. She had no petichiae or ecchymoses and her conjunctivae were pink. No lymphadenopathy was detected.

Cardiovascular examination was remarkable only for a pulse rate of 104/min. The blood pressure was 100/60 mm $\mathrm{Hg}$. Abdominal examination revealed mild right loin tenderness without any organomegaly. Clinical pelvic examination was not performed respecting the patient's

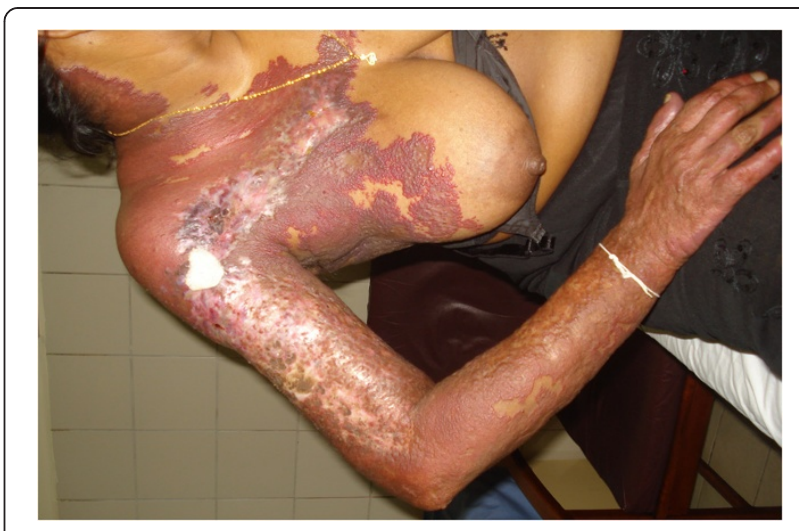

Figure 1 Non involuting hemangioma with ulcerated areas.

wishes. Respiratory system and nervous system examination were both unremarkable.

The urine full report demonstrated 15-20 pus cells and 30-40 red cells per high powered field. Complete blood count on admission showed a hemoglobin of $11 \mathrm{~g} / \mathrm{dl}$ with a neutrophil leukocytosis (WBC count of 17,000/ mm3 - neutrophils $88 \%$, lymphocytes 10\%). The platelet count was $27,000 / \mathrm{mm} 3$. C reactive protein was elevated at $196 \mathrm{mg} / \mathrm{dl}$ (reference upper limit - $6 \mathrm{mg} / \mathrm{dl}$ ). Serial complete blood counts done on days $4 \& 5$ of the illness revealed a progressive drop in platelet counts to a nadir of $15,000 / \mathrm{mm} 3$. Blood film showed severe thrombocytopenia with toxic neutrophils. Schistocytes suggestive of microangiopathic haemolytic anemia were notably absent. Her serum creatinine was mildly elevated at $1.52 \mathrm{mg} / \mathrm{dl}$ (reference upper limit - $1.3 \mathrm{mg} / \mathrm{dl}$ ). Other biochemical parameters including uric acid, electrolytes and liver profile were all within normal limits. Both blood culture and urine culture yielded gram negative Escherichia coli with identical overlapping antibiotic sensitivity patterns. The wound swab from the ulcerated areas of the hemangioma became positive for Staphylococcus aureus sensitive to both penicillin and cloxacillin. Dengue NS1 antigen was negative. Ultrasonic examination of the abdomen excluded splenomegaly, features of portal hypertension and chronic liver disease. HIV, ANA, antiphospholipid antibodies, antiplatelet antibodies and Coombs test were all negative.

Background consumptive coagulopathy was excluded with normal baseline INR, APTT, D-dimers by modified ELISA and fibrinogen levels. There was also no laboratory evidence of acute derangement of the coagulation profile in relation to the worsening of thrombocytopenias triggered by infections.

In depth perusal of her previous admission records confirmed repeated episodes of severe thrombocytopenia $(<30,000 / \mathrm{mm} 3)$ precipitated with a variety of infections with return to baseline values of approximately $100,000 / \mathrm{mm} 3$ on 
resolution of the illness (Table 1). Despite extensive investigations no cause or basis had ever been identified in her to explain the phenomenon. The last episode of severe thrombocytopenia was triggered by an upper respiratory tract infection which had occurred 4 months preceding her current illness. Community acquired

Table 1 Platelet count timeline in relation to clinical condition

\begin{tabular}{|c|c|c|}
\hline Date & $\begin{array}{l}\text { Platelet } \\
\text { count } \times 10^{3 /} \mathrm{uL}\end{array}$ & Cliinical status \\
\hline 13 February 2002 & 85 & Baseline \\
\hline 20 May 2003 & 75 & Baseline \\
\hline 01 September 2003 & 50 & Respiratory tract infection \\
\hline 08 September 2003 & 47 & $\begin{array}{l}\text { Surgery abandoned - Cannula } \\
\text { site bleed }\end{array}$ \\
\hline 10 October 2003 & 22 & Fever- Source / Cause? Viral \\
\hline 10 February 2004 & 33 & Gasteroenteritis \\
\hline 20 February 2004 & 88 & Convalescent \\
\hline 03 March 2006 & 123 & Baseline \\
\hline 01 July 2006 & 23 & Tonsillitis \\
\hline 11 July 2006 & 86 & Baseline \\
\hline 14 July 2006 & 78 & Baseline \\
\hline 07 August 2006 & 171 & Baseline \\
\hline 25 November 2006 & 38 & Viral fever \\
\hline 01 December 2006 & 88 & Convalescent \\
\hline 26 December 2006 & 86 & Baseline \\
\hline 23 January 2007 & 134 & Baseline \\
\hline 01 December 2007 & 24 & Otitis media \\
\hline 22 December 2007 & 88 & Baseline \\
\hline 10 April 2009 & 22 & Skin sepsis \\
\hline 14 April 2009 & 71 & Convalescent \\
\hline 27 April 2009 & 143 & Baseline \\
\hline 08 August 2010 & 52 & Skin sepsis \\
\hline 12 August 2010 & 72 & Baseline \\
\hline 19 August 2010 & 90 & Baseline \\
\hline 02 May 2011 & 130 & Baseline \\
\hline 02 July 2012 & 48 & Upper respiratory tract infection \\
\hline 03 July 2012 & 46 & Upper respiratory tract infection \\
\hline 04 July 2012 & 60 & Upper respiratory tract infection \\
\hline 10 August 2012 & 92 & Baseline \\
\hline 01 October 2012 & 61 & Urinary \& skin sepsis \\
\hline 02 October 2012 & 27 & Urinary \& skin sepsis \\
\hline 03 October 2012 & 15 & Urinary \& skin sepsis \\
\hline 06 October 2012 & 68 & Urinary \& skin sepsis \\
\hline 07 October 2012 & 78 & Convalescent \\
\hline 14 October 2012 & 101 & Baseline \\
\hline
\end{tabular}

infections and wound sepsis complicating ulcerated areas over the hemangioma were the commonest precipitants identified.

Bone marrow studies done during a recent episode of severe thrombocytopenia had demonstrated a normal marrow including megakaryopoiesis, whilst the peripheral blood smear had shown evidence of thrombocytopenia and toxic left shift in the neutrophils which were considered to be consistent with a peripheral cause for thrombocytopenia on a background of ongoing infection. Repeated therapeutic trials with steroids had shown no improvement in the thrombocytopenia during episodes of infection and a rise in platelet counts was always temporally related to the resolution of the infection rather than steroid therapy. The baseline thrombocytopenias were not severe enough to warrant continued steroid therapy. However the episodic thrombocytopenias with infections had initially been treated with steroids. Platlet count trends during infections showed that the thrombocytopenia seemed to improve with the resolution of the causative infection rather than steroids. This was observed on many occasions in the patient and after the initial few trials no further steroid therapy was attempted. Thrombocytopenia was seen even on several occasions where the patient presented with untreated infections where she had neither been prescribed or used any medications and hence a drug induced cause was deemed unlikely. Acetaminophen was used by the patient unrelated to infections as an analgesic especially in relation to painful menstruation and no alteration in baseline platelet counts attributable to acetaminophen were seen.

Her current illnesswas treated with a combination of intravenous levofloxacin and clindamycin which resulted in complete defervescence by the 3rd day of hospitalisation. When she was discharged on the 3rd day post admission, her platelet counts had risen to $79,000 / \mathrm{mm} 3$ with improvement in serum creatinine to $1.2 \mathrm{mg} / \mathrm{dl}$ and the urine full report was free of any evidence of persistent urinary sepsis. She was discharged on oral co amoxiclav and clindamycin for a further period of one week and advised to continue oral penicillin indefinitely to prevent wound sepsis.

\section{Discussion}

Congenital hemangiomas are benign abnormal proliferation of blood vessels [1]. Hemangiomas arise from mesenchymal tissue composed of multiple vascular channels lined with a single layer of endothelium on a scaffolding of fibrous connective tissuewhich frequently proliferate early in life before regressing in size. Vascular malformations despite many similarities in architecture to hemangiomas, differ by the presence of dysplastic endothelium and lack of regression. Congenital hemangiomas despite being present at birth often become clinically evident only later. Vascular 
malformations are named after the vascular element they most closely resemble namely capillary, venous, and lymphatic [13]. Rapidly involuting ( $\mathrm{RICH}$ ) and non-involuting (NICH) "congenital" hemangiomas appear to be histopathological hybrids showing features of both vascular tumor and malformation, and both entities are believed to lie within the same spectrum [14].

Noninvoluting congenital hemangiomas are rare variants of cutaneous vascular tumors of intrauterine onset without sex prevalence and they are described as wellcircumscribed, plaque-like or slightly bossed lesions which never disappear and persist either unchanged or with slight expansion [2].

In a case series by Jin et al. magnetic resonance imaging, computed tomographic angiography and digital subtraction angiography of non involuting congenital hemangioma gave findings similar to radiological findings of common infantile hemangiomas [15].

The platelet count in adults ranges from 150,000 to $450,000 / \mathrm{mm} 3$, with mean values of 237,000 and $266,000 / \mathrm{mm} 3$ in males and females, respectively [16]. Thrombocytopenia is defined as a platelet count of less than $150,000 / \mathrm{mm} 3$. Thrombocytopenia may be caused by increased peripheral destruction, sequestration or reduced production. A detailed description of the causes thrombocytopenia is not within the scope of this case report, as a multitude of causes including many states of infection could be possible aetiologies [6].

Clinical observation of repeated episodes of severe thrombocytopenia during a succession of varieties of infections temporally establishes the relationship between the phenomenon of severe thrombocytopenia and infections in this patient The authors excluded common and likely alternative causes of thrombocytopenia in the subject including dengue fever, drugs, portal hypertension and marrow pathologies and therefore the phenomenon is explained by either sequestration or increased destruction within the hemangioma which may either be immune or non immune [6]. This is further supported by the combined findings of the blood film and bone marrow trephine biopsy done during a similar episode in the past which showed thrombocytopenia and normal megakaryopoiesis respectively. The chronic thrombocytopenia is likely due to due to chronic consumption within the hemangioma. A baseline state of inflammation within the hemangioma with aggravation due to infections causing increased consumption could be postulated however demonstrating or disproving this requires molecular and microscopic studies which are not feasible for ethical reasons. Sequestration causing thrombocytopenia is also unlikely as an explanation for the acute thrombocytopenia with infection though such an explanation cannot be discounted as the reason for the baseline state of low platelets. Radiolabelled platelet tracer studies are not possible in the clinical setting where the authors encountered the patient.

In patients without hemangiomas chronic immune thrombocytopenia is known to occur in infections [7], and acute immune mediated thrombocytopenia has been demonstrated in certain infections and hypersensitivity states [8-11]. Thrombocytopenia associated with infections has been known to parallel the severity of illness [12]. It was postulated that this patient who had a baseline thrombocytopenia due to sequestration and destruction within the hemangioma most likely developed superimposed immune mediated destruction of platelets triggered by a variety of infective conditions.

Hemangioma-thrombocytopenia syndrome associated with microangiopathic hemolytic anemia has been documented in children. The Kasabach-Merritt phenomenon was first described in 1940 as a syndrome of consumptive coagulopathy, with capillary hemangiomas having clearly demonstrable microangiopathic haemolyic anemia and thrombocytopenia [3]. Rapidly involuting congenital haemangioma is known to be associated with transient thrombocytopenia and coagulopathy [4]. Although thrombocytopenia has been documented in adult patients with large or multiple hemangiomas s no reports were found in literature documenting repeated episodes of severe thrombocytopenia triggered by a variety of successive infectious conditions in a patient having a non involuting congenital hemangioma with baseline thrombocytopenia.

\section{Conclusion}

The authors report this case as a novel observation and suggest that further studies are needed to establish the phenomenon and pathogenesis in cohorts with non involuting congenital hemangiomas.

\section{Consent}

Written informed consent was obtained from the patient for publication of this case report and any accompanying images. A copy of the written consent is available for review by the editor of this journal.

\section{Competing interests}

The author(s) declare that they have no competing interests.

\section{Authors' contributions}

All authors were involved in the clinical management of the patient, literature survey and drafting of the manuscript. All authors read and approved the final manuscript.

\section{Author details}

${ }^{1}$ National Hospital of Sri Lanka, Regent Street, Colombo 7, Sri Lanka. ${ }^{2}$ Lanka Hospitals, Colombo 5, Sri Lanka. ${ }^{3}$ Faculty of Medicine, University of Sri Jayawardenapura, Nugegoda, Sri Lanka.

Received: 18 October 2012 Accepted: 11 June 2013

Published: 13 June 2013 


\section{References}

1. Bruckner AL, Frieden IJ: Hemangiomas of infancy. J Am Acad Dermatol 2003, 48(4):477-493

2. Enjolras O, Mulliken JB, Boon LM, Wassef M, Kozakewich HP, Burrows PE: Noninvoluting congenital hemangioma: a rare cutaneous vascular anomaly. Plast Reconstr Surg 2001, 107(7):1647-1654.

3. Propp RP, Scharfman WB: Hemangioma-thrombocytopenia syndrome associated with microangiopathic hemolytic anemia. Blood 1966, 28(5):623-633.

4. Baselga E, Cordisco MR, Garzon M, Lee MT, Alomar A, Blei F: Rapidly involuting congenital haemangioma associated with transient thrombocytopenia and coagulopathy: a case series. Br J Dermatol 2008, 158(6):1363-1370.

5. Lang PG, Dubin HV: Hemangioma-thrombocytopenia syndrome; a disseminated intravascular coagulopathy. Arch Dermatol 1975, 111(1):105-107.

6. Sekhon SS, Roy V: Thrombocytopenia in adults: A practical approach to evaluation and management. South Med J 2006, 99(5):491-498.

7. Stasi R, Willis F, Shannon MS, Gordon-Smith EC: Infectious causes of chronic immune thrombocytopenia. Hematol Oncol Clin North Am 2009, 23(6):1275-1297.

8. Bautista MA, Stevens WT, Chen CS, Curtis BR, Aster RH, Hsueh CT: Hypersensitivity reaction and acute immune-mediated thrombocytopenia from oxaliplatin: two case reports and a review of the literature. J Hematol Oncol 2010, 3:12.

9. Namdev R, Dutta SR, Singh H: Acute immune thrombocytopenic purpura triggered by insect bite. J Indian Soc Pedod Prev Dent 2009, 27(1):58-61.

10. Cines DB, Liebman HA: The immune thrombocytopenia syndrome: a disorder of diverse pathogenesis and clinical presentation. Hematol Oncol Clin North Am 2009, 23(6):1155-1161.

11. Cines DB, Liebman H, Stasi R: Pathobiology of secondary immune thrombocytopenia. Semin Hematol 2009, 46(1 Suppl 2):S2-S14.

12. Stasi R: Immune thrombocytopenia: pathophysiologic and clinical update. Semin Thromb Hemost 2012, 38(5):454-462.

13. Elsayes KM, Menias CO, Dillman JR, Platt JF, Willatt JM, Heiken JP: Vascular malformation and hemangiomatosis syndromes: spectrum of imaging manifestations. AJR Am J Roentgenol 2008, 190(5):1291-1299.

14. Mulliken JB, Enjolras $\mathrm{O}$ : Congenital hemangiomas and infantile hemangioma: missing links. J Am Acad Dermatol 2004, 50(6):875-882.

15. Jin $Y B$, Lin XX, Ma G, Chen H, Li W, Fan XD, Lin MS, Wang W, Zhou ZP. Non-involuting congenital hemangioma: a study for diagnosis and treatment. Zhonghua Zheng Xing Wai Ke Za Zhi 2009, 25(3):189-193.

16. Buckley MF, James JW, Brown DE, Whyte GS, Dean MG, Chesterman CN, Donald JA: A novel approach to the assessment of variations in the human platelet count. Thromb Haemost 2000, 83(3):480-484.

doi:10.1186/2052-1839-13-7

Cite this article as: Shivanthan et al:: Baseline thrombocytopenia complicated by recurrent episodes of transient severe thrombocytopenia following infections in an adult woman with a non involuting congenital hemangioma - a case report. BMC Hematology 2013 13:7.

\section{Submit your next manuscript to BioMed Central and take full advantage of:}

- Convenient online submission

- Thorough peer review

- No space constraints or color figure charges

- Immediate publication on acceptance

- Inclusion in PubMed, CAS, Scopus and Google Scholar

- Research which is freely available for redistribution 